\title{
Cytotoxicity, cell cycle arrest, and apoptosis in breast cancer cell lines exposed to an extract of the seed kernel of Mangifera pajang (bambangan).
}

\begin{abstract}
An extract of Mangifera pajang kernel has been previously found to contain a high content of antioxidant phytochemicals. The present research was conducted to investigate the anticancer potential of this kernel extract. The results showed that the kernel crude extract induced cytotoxicity in MCF-7 (hormone-dependent breast cancer) cells and MDA-MB-231 (nonhormone dependent breast cancer) cells with IC50 values of 23 and $30.5 \mu \mathrm{g} / \mathrm{ml}$, respectively. The kernel extract induced cell cycle arrest in MCF-7 cells at the sub-G1 (apoptosis) phase of the cell cycle in a time-dependent manner. For MDA-MB-231 cells, the kernel extract induced strong G2-M arrest in cell cycle progression at 24. h, resulting in substantial sub-G1 (apoptosis) arrest after 48 and 72. h of incubation. Staining with Annexin V-FITC and propidium iodide revealed that this apoptosis occurred early in both cell types, $36 \mathrm{~h}$ for MCF-7 cells and 24. h for MDA-MB-231cells, with $14.0 \%$ and $16.5 \%$ of the cells respectively undergoing apoptosis at these times. This apoptosis appeared to be dependent on caspase-2 and -3 in MCF-7 cells, and on caspase-2, -3 and -9 in MDA-MB-231 cells. These findings suggest that $\mathrm{M}$. pajang kernel extract has potential as a potent cytotoxic agent against breast cancer cell lines.
\end{abstract}

Keyword: Apoptosis; Breast cancer; Cytotoxicity; Mangifera pajang. 